\title{
Spin Filtering of Free Electrons by Magnetic Multilayers: Towards an Efficient Self-Calibrated Spin Polarimeter
}

\author{
C. Cacho, ${ }^{1, *}$ Y. Lassailly, ${ }^{1,2}$ H.-J. Drouhin, ${ }^{1}$ G. Lampel, ${ }^{1}$ and J. Peretti ${ }^{1}$ \\ ${ }^{1}$ Laboratoire de Physique de la Matière Condensée (UMR 7643-CNRS), Ecole Polytechnique, F-91128 Palaiseau, France \\ ${ }^{2}$ Department of Physics, California Institute of Technology, Pasadena, California 91125
}

(Received 10 August 2001; published 23 January 2002)

\begin{abstract}
An asymmetrical ferromagnetic cobalt bilayer $(18 \mathrm{~nm} \mathrm{Au} / 0.8 \mathrm{~nm} \mathrm{Co} / 2.2 \mathrm{~nm} \mathrm{Au} / 1.3 \mathrm{~nm} \mathrm{Co} / 1.5 \mathrm{~nm}$ $\mathrm{Au}$ ) operates as a self-calibrated spin polarimeter with a high spin selectivity for free electrons injected at a few $\mathrm{eV}$ above the Fermi level. We present the analysis of transmitted currents as a function of the incident energy, based on a model of spin polarization dilution into the first gold layer and ballistic transport close to the vacuum level throughout the sample.
\end{abstract}

DOI: 10.1103/PhysRevLett.88.066601

PACS numbers: 72.15.Lh, 75.70. $-\mathrm{i}, 75.90 .+\mathrm{w}$

Since the Stern and Gerlach experiment, spin filtering has been demonstrated in systems associating ferromagnetic and nonmagnetic materials. One of its most spectacular manifestations is the giant magnetoresistance in ferromagnetic multilayers [1]. It refers to the conductivity at the Fermi level, which is strongly correlated to the orientation of magnetization. In contrast, spin polarized electron spectroscopy offers the opportunity to address spin-dependent transport of ballistic electrons traveling at energies of a few tens of $\mathrm{eV}$ above the Fermi level. In order to do so, one can examine the transmission of an unpolarized electron beam from a nonmagnetic substrate through an ultrathin 3D ferromagnet $[2,3]$. Unpolarized electrons are then found to become polarized after traversing the magnetic layer. This so-called overlayer technique shows the preferential emission of a spin direction from the ferromagnetic layer, defining an electron spin filter effect. The spin polarization can be explained by the electron inelastic scattering involving unoccupied $d$ states above the Fermi level $[4,5]$. The scattering rate for minority spin electrons is then enhanced with respect to that of majority spin electrons due to the excess of minority spin holes.

The first attempts to characterize electron spin filtering well above the Fermi level were attained by measuring the direct transmission of a spin polarized free electron beam through a freestanding $\mathrm{Au} / \mathrm{Co} / \mathrm{Au}$ film [6,7]. It was shown that the transmitted current depends on the relative orientation of the incident spin polarization with respect to the cobalt layer magnetization. This approach offers the advantages of tuning the energy and the spin polarization of the incoming electrons, and of separating the inelastic and the elastic contributions to the transmitted current. By analogy to spin polarimetry, an ultrathin ferromagnetic layer can be described by its spin discriminating power, the so-called Sherman function $s$. It is the polarization acquired by an unpolarized beam when passed through the spin filter [8]. The $\mathrm{Au} / 1 \mathrm{~nm} \mathrm{Co} / \mathrm{Au}$ structure, which has an $s$ value of 0.34, behaves as an electron spin detector [9].

In this Letter, we show that the $\mathrm{Au} / \mathrm{Co} / \mathrm{Au} / \mathrm{Co} / \mathrm{Au}$ cobalt bilayer constitutes a self-calibrated spin polarimeter.
Two uncoupled ferromagnetic layers of different thickness $d_{1}$ and $d_{2}$ have different coercive fields. Therefore a bilayer structure can be magnetized in four saturated configurations: two of them, that we call " $F$ " and " $F$ " correspond to parallel saturated magnetizations in both layers, and the other two labeled "AF" and "-AF" refer to antiparallel saturated magnetizations. The two reversed configurations $\mathrm{F}(\mathrm{AF})$ and $-\mathrm{F}(-\mathrm{AF})$ are equivalent for the transmission of an unpolarized beam, whereas they yield different transmissions for a polarized beam. It is therefore possible to perform three separated measurements: the transmission asymmetry of an unpolarized beam between $\mathrm{F}$ and $\mathrm{AF}$ configurations and the transmission asymmetries of a polarized beam between $\mathrm{F}$ and $-\mathrm{F}$ and between $\mathrm{AF}$ and - AF configurations. As a consequence, one obtains separately three quantities: the spin discriminating power $s_{1}$ and $s_{2}$ of each layer and the polarization $P_{0}$ of the incident beam. Furthermore, from the microscopic analysis of $s_{1}$ and $s_{2}$ in terms of spin-dependent inelastic scattering at the interfaces and in the bulk of the ferromagnetic layers, we determine a characteristic spin-discriminating length in bulk cobalt and the spin-filtering power of $\mathrm{Au} / \mathrm{Co}$ interfaces. We also show evidence that spin transport above the Fermi level is mainly governed by the creation of a low energy secondary electrons distribution, followed by a ballistic transmission at an energy close to the vacuum level. The formation of the electron distribution leads to a dilution of the polarization by secondary electrons.

The transmission experiment consists of focusing a longitudinally polarized electron beam photoemitted from a GaAs source on a cobalt bilayer with perpendicular magnetization $(18 \mathrm{~nm} \mathrm{Au} / 0.8 \mathrm{~nm} \mathrm{Co} / 2.2 \mathrm{~nm} \mathrm{Au} / 1.3 \mathrm{~nm} \mathrm{Co} /$ $1.5 \mathrm{~nm} \mathrm{Au})$. The spin polarization $P_{0}$ of the incident beam lies along the direction of the sample magnetization. Experimental details on sample preparation can be found in Ref. [9]. The sample is magnetized in situ by pulses of the magnetic field, which allows one to select the four distinct states of saturated magnetization. The electron beam enters the thickest gold side. The low energy injection threshold is given by the vacuum level of the entrance side, whereas 
the lowest possible energy of the transmitted electron is the vacuum level $E_{V}$ of the exit side of the cap Au layer. The vacuum level of both gold faces was lowered at best by $3 \mathrm{eV}$ by cesium deposition. The electrons emerging from the multilayer build up an energy distribution curve, which includes, besides the elastic electrons, a distribution of inelastically scattered electrons. A retarding-field analyzer with an energy resolution of $0.2 \mathrm{eV}$ FWHM allows one to separate these two parts. The inelastic contribution is located at the energy $E_{V}$ and originates from electrons having lost energy and from secondary electrons excited from the Fermi sea. In the following, we will discuss only the inelastic part of transmitted beam, which is in our case essentially responsible for the spin-dependent transport above $E_{F}[9]$.

The transmitted currents $I_{P}^{\mu}$ are measured at $E_{V}$, where $\mu$ stands for the bilayer magnetization configuration and $P$ for the polarization values $\left(0\right.$ or $\left.\pm P_{0}\right)$. We separate $I_{P}^{\mu}$ into its spin-independent contribution $I_{0}^{\mu}$ and its spindependent contribution $\Delta I_{P}^{\mu}, I_{P}^{\mu}=I_{0}^{\mu}+\Delta I_{P}^{\mu} . I_{0}^{\mu}$ is actually the transmitted current for an unpolarized beam. Let us remark that changing $P$ into $-P$ is equivalent to changing $\mu$ into $-\mu$, so that $I_{0}^{\mu}=\frac{1}{2}\left(I_{P_{0}}^{\mu}+I_{-P_{0}}^{\mu}\right)=\frac{1}{2}\left(I_{P_{0}}^{\mu}+I_{P_{0}}^{-\mu}\right)$ and $\Delta I_{P_{0}}^{\mu}=\frac{1}{2}\left(I_{P_{0}}^{\mu}-I_{-P_{0}}^{\mu}\right)=\frac{1}{2}\left(I_{P_{0}}^{\mu}-I_{P_{0}}^{-\mu}\right)=I_{P_{0}}^{\mu}-I_{0}^{\mu}$.

First, when injecting an unpolarized electron beam $(P=0)$ at very low incident energy $E_{\text {inc }}=3.4 \mathrm{eV}$ (referred to the Fermi level $E_{F}$ of the sample), we measure the transmitted current $I_{0}^{\mu}$ at $E_{V}=1.7 \mathrm{eV}$ versus pulse amplitudes of the magnetic field [diamonds in Fig. 1(a)]. Such a measurement is equivalent to complete the hysteresis cycle of the sample. The largest transmissions are reached at high magnetic fields in the configurations

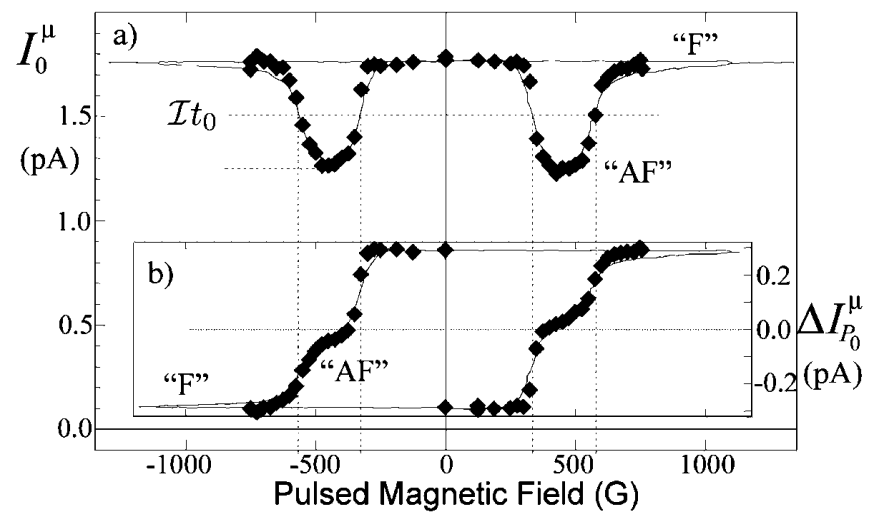

FIG. 1. (a) Transmitted current $I_{0}^{\mu}$ of an unpolarized beam injected at $3.4 \mathrm{eV}$ above the sample's Fermi level versus magnetic field $(\diamond)$. The vacuum level of the exit face $E_{V}$ is lowered to $1.7 \mathrm{eV}$. Corresponding fit calculated from Eq. (2) (solid line), I $t_{0}$ being the transmitted current when one Co layer is demagnetized (horizontal dotted line). (b) Spin-dependent transmitted current $\Delta I_{P_{0}}^{\mu}$ versus magnetic field ( $\bullet$ ). Scaled magneto-optical effect hysteresis loop showing the " $F$ " and "AF" magnetization states (solid line). Vertical lines visualize the coercive fields of each Co layer. Both current measurements are normalized to $100 \mathrm{nA}$ of incident current. identified as $\mathrm{F}$ and $-\mathrm{F}$ and sharply drop by about $30 \%$ when the bilayer is set to the AF or - AF configurations. This transmitted asymmetry of an unpolarized electron beam between the parallel and antiparallel magnetization configurations is analogous to the spin-valve effect (or polarizing-analyzing effect) present in spin-valve transistors $[10,11]$ and ballistic electron emission miscroscopy experiments [12]. The first cobalt layer polarizes the beam by passing a spin direction, whereas the second layer detects the projected spin component. Conditions of total extinction will be defined below.

Second, working with an incident beam of polarization $P=P_{0}$, the relevant quantity is $\Delta I_{P_{0}}^{\mu}$, which is reported on Fig. 1(b) versus the pulsed magnetic field. $\Delta I_{P_{0}}^{\mu}$ is directly measured by modulating the incident polarization between $P_{0}$ and $-P_{0}$ and using a lock-in amplifier. $\Delta I_{P_{0}}^{\mu}$ reproduces the magnetization cycle of the sample. One can easily identify the four magnetization states $\mathrm{F},-\mathrm{F}, \mathrm{AF}$, and $-\mathrm{AF}$.

To describe quantitatively the transmission above $E_{F}$, we propose the following model. At very low $E_{\text {inc }}$, the electron distribution propagates ballistically within an energy range close to $E_{V}$ by following an exponential attenuation versus distance. In this transport regime, no spin relaxation in the cobalt layers and in the $2.2 \mathrm{~nm}$ thick gold layer is assumed. At low energy, the electron inelastic mean-free path is large, i.e., of the same order as the cobalt layer thickness. Magnetization resulting from the distribution of opposite domains can be described by the respective domain fractions $\frac{1}{2}\left(1 \pm \eta_{l}\right)$, with $l$ indexing the cobalt layers and $\eta_{l}$ varying continuously from -1 to 1 . We define $I$ as the current entering the first spin filter and $\mathcal{P}$ its polarization. From the formalism of spin polarimetry $[8,13,14]$ applied to the bilayer, the transmitted current is

$$
I_{P}^{\mu}=I t_{0}\left(1+\eta_{1} \eta_{2} s_{1} s_{2}+\mathcal{P}\left(\eta_{1} s_{1}+\eta_{2} s_{2}\right)\right),
$$

where $\eta_{1} s_{1}$ and $\eta_{2} s_{2}$ are the Sherman functions of the Co layer (analyzer), and $t_{0}$ is the product of the spin-average transmissions of the four layers from the first magnetic layer to the exit surface. From Eq. (1), one obtains

$$
I_{0}^{\mu}=I t_{0}\left(1+\eta_{1} \eta_{2} s_{1} s_{2}\right),
$$

and

$$
\Delta I_{P_{0}}^{\mu}=I \mathcal{P} t_{0}\left(\eta_{1} s_{1}+\eta_{2} s_{2}\right) .
$$

Note that (2) implies that (i) a perfect spin-valve effect $\left(s_{1}=s_{2}=1\right)$ would yield a zero transmitted current $I_{0}^{\mu}$ in $\mathrm{AF}$ states $\left(\eta_{1} \eta_{2}=-1\right)$ (extinction condition), and (ii) $I t_{0}$ is the transmitted current, for an unpolarized electron beam, when at least one of the Co layers is demagnetized $\left(\eta_{1}\right.$ or $\left.\eta_{2}=0\right)$. In Fig. 1, the corresponding magnetization states are indicated by vertical dotted lines set at the coercive fields of the two Co layers. The 
$I_{0}^{\mu}$ values associated with these states are the averaged transmitted currents between the $\mathrm{F}$ and the AF states $\frac{1}{2}\left(I_{0}^{\mathrm{F}}+I_{0}^{\mathrm{AF}}\right)=I t_{0}$.

By using $\eta_{1}$ and $\eta_{2}$ values extracted from the hysteresis loop associated with each Co layer, Eq. (2) successfully fits the step variation of $I_{0}^{\mu}$ [solid lines in Fig. 1(a)]. For an unpolarized incident beam $(P=0)$, the asymmetry $A_{I_{0}}$ in transmitted currents between $\mathrm{F}$ and $\mathrm{AF}$ states, deduced from Fig. 1(a), yields directly the value of $s_{1} s_{2}=A_{I_{0}}=$ $\left(I_{0}^{\mathrm{F}}-I_{0}^{\mathrm{AF}}\right) /\left(I_{0}^{\mathrm{F}}+I_{0}^{\mathrm{AF}}\right)=0.17 \pm 0.02$. On the other hand, for a spin polarized incident beam $\left(P=P_{0}\right)$, the relevant asymmetry $A_{\Delta I_{P_{0}}}$ between the $\mathrm{F}$ and the AF states calculated from Fig. 1(b) gives the ratio $s_{1} / s_{2}=A_{\Delta I_{P_{0}}}=$ $\left(\Delta I_{P_{0}}^{\mathrm{F}}-\Delta I_{P_{0}}^{\mathrm{AF}}\right) /\left(\Delta I_{P_{0}}^{\mathrm{F}}+\Delta I_{P_{0}}^{\mathrm{AF}}\right)=0.70 \pm 0.03$. Obviously, this nonunity value expresses that in the $\mathrm{AF}$ configurations the saturated magnetizations of the two layers do not compensate, which is expected since both Co layers have different thicknesses. The spin selectivities of the two layers deduced from the above values of $s_{1} s_{2}$ and $s_{1} / s_{2}$ are $s_{1}=0.34 \pm 0.02$ and $s_{2}=0.49 \pm 0.03$. By analogy with usual spin polarimetry, we write

$$
A_{I_{P_{0}}}=\left(I_{P_{0}}^{\mathrm{F}}-I_{P_{0}}^{-\mathrm{F}}\right) /\left(I_{P_{0}}^{\mathrm{F}}+I_{P_{0}}^{-\mathrm{F}}\right)=s^{F} \mathcal{P},
$$

which defines the Sherman function of the bilayer in the $\mathrm{F}$ state [8]. From the previous definition of $I_{0}^{\mu}$ and $\Delta I_{P_{0}}^{\mu}, A_{I_{P_{0}}}$ is equal to $\Delta I_{P_{0}}^{\mathrm{F}} / I_{0}^{\mathrm{F}}=0.17 \pm 0.02$ and, using Eqs. (2) and $(3), s^{\mathrm{F}}=\left(s_{1}+s_{2}\right) /\left(1+s_{1} s_{2}\right)=0.7 \pm 0.04$. From Eq. (4), we then obtain $\mathcal{P}=0.24 \pm 0.02$, which is precisely the value of the polarization $P_{0}$ produced by the GaAs source that we use. This result shows that a calibration of the initial spin polarization is achieved and also validates the ballistic transport assumption at very low $E_{\text {inc }}$.

In order to verify the ballistic transport assumption at higher $E_{\text {inc }}$, we investigate the variation of $I_{0}^{\mu}$ relative to $E_{\text {inc }}$ restricting ourselves to the $\mathrm{F}$ and $\mathrm{AF}$ states. In this experiment, the work function of the exit face has raised up to $E_{\text {inc }}=2.5 \mathrm{eV}$. The net increase in $I_{0}^{\mu}$ reveals the creation of secondary electrons, as seen on Fig. 2(a). As expected, the transmission is much higher in the $\mathrm{F}$ state. When $E_{\text {inc }} \gg E_{V}$, the central result is that the asymmetry $A_{I_{0}}=0.12$ does not depend upon $E_{\text {inc }}$ [Fig. 2(a)]. Since $s$ is a decreasing function of the transport energy inside the spin filter [15], this invariance shows that transport in the ferromagnetic layers takes place at the same energy. The subjacent model is the creation of secondary electrons in the first atomic gold layers followed by ballistic propagation at energy close to $E_{V}$ across the Co layers [16]. Consequently, according to (2) the current $I$ entering the first cobalt layer increases almost linearly as a function of $E_{\text {inc }}$. Since secondary electrons are generated into the first Au layer, the initial polarization $P_{0}$ should be diluted by unpolarized secondary electrons excited from the Au conduction band. A first hint is given by the measurement of $\Delta I_{P_{0}}^{\mu}$, which is constant [see Fig. 2(b)]. This

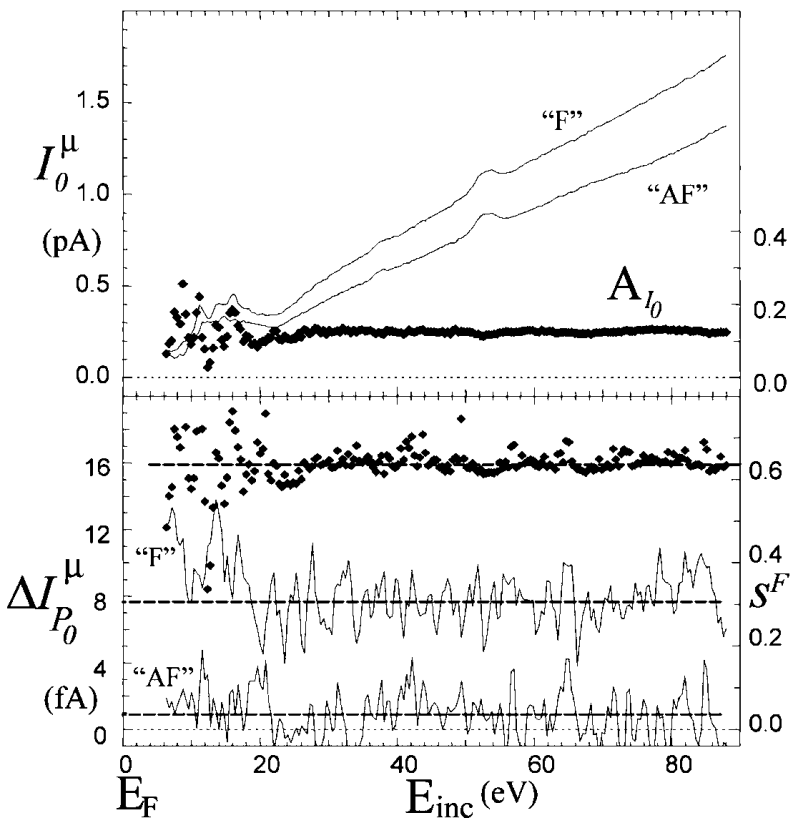

FIG. 2. (a) Inelastically transmitted currents $I_{0}^{\mathrm{F}}$ and $I_{0}^{\mathrm{AF}}$ in the $\mathrm{F}$ and $\mathrm{AF}$ configurations (left scale) and the asymmetry $A_{I_{0}}$ measured versus the incident beam energy $E_{\text {inc }}$ (right scale). (b) Difference in transmitted currents between $P_{0}$ and 0 spin polarized electron beam for the $\mathrm{F}$ and $\mathrm{AF}$ configurations (left scale) and the spin filter selectivity $s^{\mathrm{F}} . E_{\text {inc }}=0$ corresponds to the sample's Fermi level. Dotted lines give the averaged values. Data recorded for $E_{V}=2.5 \mathrm{eV}$.

means that $I \mathcal{P}$ is constant and therefore implies that $\mathcal{P}$ is a decreasing function of $E_{\text {inc }}$ ( $t_{0}$ is constant since the ballistic transport occurs at the same energy $E_{V}$ ). To determine accurately this variation, we first calculate $s^{\mathrm{F}}$ from the measured asymmetries $A_{I_{0}}$ and $A_{\Delta I_{P_{0}}}$. Figure 2(b) displays $s^{\mathrm{F}}$ as a constant $s^{\mathrm{F}}=0.62 \pm 0.05$, a lower averaged value than the previous one, since at $E_{V}=2.5 \mathrm{eV}$ electrons travel farther above the Co $d$ bands. Figure 3 shows the polarization $\mathcal{P}=A_{I_{P_{0}}} / s^{\mathrm{F}}$ versus $E_{\text {inc }}$. The sharp fall is a direct evidence of the polarization dilution. The decrease is well reproduced by the following relation $\mathcal{P}=P_{0} E_{V} / E_{\text {inc }}$, which asserts that the initial polarization $P_{0}$ is diluted by the multiplication factor $E_{\text {inc }} / E_{V}$ produced by secondary electrons. This ratio can be derived from a model, in which $\delta$-like $d$ bands are considered at the Fermi level $[14,17]$. At each collision, an electron loses half of its energy by exciting a secondary electron. The mechanism of energy loss iterates until $E_{V}$ is reached. This model is valid as long as $E_{V}$ and the width of the $d$ bands are negligible relative to $E_{\text {inc }}$. The data fit, excluding the measurements at low $E_{\mathrm{inc}}$ given by the polarization dilution model, is plotted in Fig. 3. The extrapolation of the fit to $E_{\text {inc }}=E_{V}$ gives again $\mathcal{P}=0.24=P_{0}$.

Coming back to the microscopic analysis of $s_{1}$ and $s_{2}$, we introduce the spin-discriminating length $\delta$ and the average inelastic mean-free path in cobalt $\lambda$ by $1 / \delta=$ $\left(1 / \lambda_{-}-1 / \lambda_{+}\right) / 2$ and $1 / \lambda=\left(1 / \lambda_{-}+1 / \lambda_{+}\right) / 2$, where 


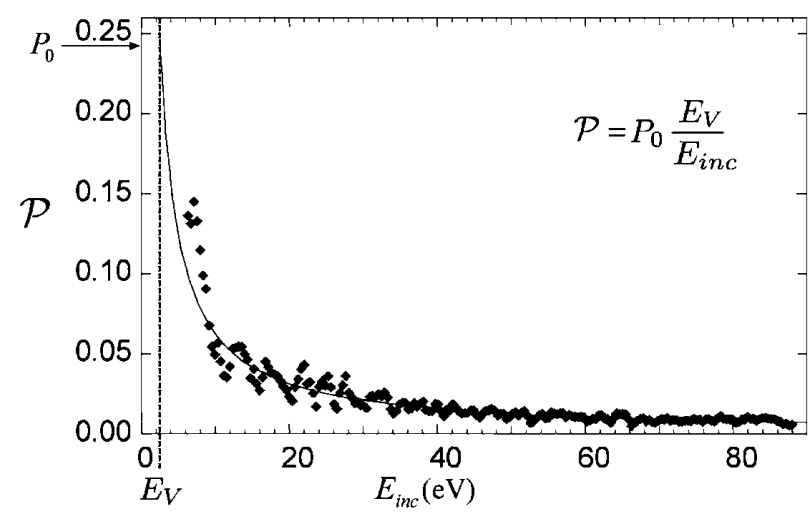

FIG. 3. Variation of the diluted polarization $\mathcal{P}$ versus the incident energy $E_{\text {inc }}$. The polarization dilution law $\mathcal{P}=P_{0} E_{V} / E_{\text {inc }}$ is drawn as a solid line. By extrapolating at $E_{\text {inc }}=E_{V}$, the initial polarization $P_{0}$ is retrieved.

$\lambda_{+}\left(\lambda_{-}\right)$is the inelastic mean-free path in cobalt for majority (minority) spins. For each of the two layers $l$, we separate the bulk and interface discriminating powers, $s_{b}$ and $s_{i}$ defined in terms of transmission asymmetries as in [13]. Then, $s_{l b}=\left(t_{+}-t_{-}\right) /\left(t_{+}+t_{-}\right)=\operatorname{th}\left(d_{l} / \delta\right)=$ $\operatorname{th}\left(a_{b} d_{l} / \lambda\right)$ and $s_{i}=\operatorname{th}\left(2 a_{i}\right)$, where $t_{ \pm}=\exp \left(-d_{l} / \lambda_{ \pm}\right)$, $a_{b}=\lambda / \delta$ is the intrinsic spin-discriminating factor in cobalt, and $a_{i}$ is the intrinsic spin-discriminating factor of one $\mathrm{Co} / \mathrm{Au}$ interface. Then, the total Sherman function for the layer $l$ is

$$
s_{l}=\left(s_{i}+s_{l b}\right) /\left(1+s_{i} s_{l b}\right)=\operatorname{th}\left(2 a_{i}+a_{b} d_{l} / \lambda\right) .
$$

The Sherman function depends on the intrinsic quantities of the magnetic material $a_{i}$ and $a_{b}$ and on the reduced thickness $d_{l} / \lambda$. Using the values of $s_{1}=0.34, s_{2}=0.49$, $d_{1}=0.8 \mathrm{~nm}$, and $d_{2}=1.3 \mathrm{~nm}$, one obtains the following from Eq. (5): $\delta=2.7 \mathrm{~nm}$ and $2 a_{i}=0.06$. The value of $\delta$, at $1.7 \mathrm{eV}$ above the Fermi level, is in good agreement with the values previously reported [12]. The comparison of the value of $2 a_{i}$ with $d_{1} / \delta=0.30$ and $d_{2} / \delta=0.48$ shows that the interface effect is much smaller than the bulk effect (it is equivalent to the bulk effect in a layer of thickness of $0.16 \mathrm{~nm}$ ). The contribution of the two interfaces to the Sherman function is in fact of the order of the experimental uncertainty, since $s_{1}, s_{2}, d_{1}$, and $d_{2}$ are not known with an accuracy better than $5 \%$.

In conclusion, the analysis of the transmission through a ferromagnetic bilayer of free spin polarized electrons provides the physical quantities, which describe the spindependent transport: the spin-discriminating length in the ferromagnet, the Sherman function of the two layers, and the bulk and interface contributions to the spin-filtering effect. Finally, the incident beam polarization $P_{0}$ is measured without the need for any external calibration and without relying upon any microscopic model of spindependent transport. Multilayered ferromagnetic structures open up the way to new self-calibrated electron spin polarimeters with high selectivity.

The authors are indebted to E. Seddon for her helpful and substantial discussions and P. Seneor for his critical reading of the manuscript.

*Electronic address: c.cacho@dl.ac.uk

[1] M. Baibich, J. Broto, A. Fert, F. Nguyen Van Dau, F. Petroff, P. Etienne, G. Creuzet, A. Friederich, and J. Chazelas, Phys. Rev. Lett. 61, 2472 (1988).

[2] D. P. Pappas, K. P. Kamper, B. P. Miller, H. Hopster, D. E. Fowler, C. R. Brundle, A. C. Luntz, and Z. X. Shen, Phys. Rev. Lett. 66, 504 (1991).

[3] J. C. Grobli, D. Guarisco, S. Frank, and F. Meier, Phys. Rev. B 51, 2945 (1995).

[4] G. Schonhense and H. C. Siegmann, Ann. Phys. (Leipzig) 2, 465 (1993).

[5] H. J. Drouhin, Phys. Rev. B 56, 14886 (1997).

[6] D. Oberli, R. Burgermeister, S. Riesen, W. Weber, and H. C. Siegmann, Phys. Rev. Lett. 81, 4228 (1998).

[7] Y. Lassailly, H. Drouhin, A. van der Sluijs, G. Lampel, and C. Marliere, Phys. Rev. B 50, 13054 (1994).

[8] J. Kessler, Polarized Electrons (Springer-Verlag, Berlin, 1976).

[9] H. J. Drouhin, A. M. Van der Sluijs, Y. Lassailly, and G. Lampel, J. Appl. Phys. 79, 4734 (1996).

[10] D. Monsma, J. Lodder, T. Popma, and B. Dieny, Phys. Rev. Lett. 74, 5260 (1995).

[11] R. Jansen, P. Anil Kumar, O. van't Erde Haan, and J. Lodder, Phys. Rev. Lett. 85, 3277 (2000).

[12] W. H. Rippard and R. Buhrman, Phys. Rev. Lett. 84, 971 (2000).

[13] A generalized definition of the Sherman function of a spin filter of transmission $t_{+}\left(t_{-}\right)$is $s=\left(t_{+}-t_{-}\right) /\left(t_{+}+t_{-}\right)$, and the transmission of an electron beam with a polarization $P$ is given by $t(1+s P)$, where $t$ is the mean transmission $\left(t_{+}+t_{-}\right) / 2$.

[14] A. Filipe, H. J. Drouhin, G. Lampel, Y. Lassailly, J. Nagle, J. Peretti, and A. Schuhl, Phys. Rev. Lett. 80, 2425 (1998).

[15] C. Cacho, Ph.D. thesis, Ecole Polytechnique, 2000.

[16] It was previously found that the transmission through a single spin filter $(18 \mathrm{~nm} \mathrm{Au} / 1 \mathrm{~nm} \mathrm{Co} / 2 \mathrm{~nm} \mathrm{Au})$ is not modified when turning the sample over.

[17] D. R. Penn, S. P. Apell, and S. M. Girvin, Phys. Rev. B 32, 7753 (1985). 